\title{
TTR
}

Traduction, terminologie, re?daction

\section{Katharina Reiss. La critique des traductions, ses possibilités et ses limites, traduit de l'allemand par Catherine Bocquet, Cahiers de l'Université d'Artois 23/2002, Arras, Artois Presses Université, 2002, 166 p.}

\section{Claude Tatilon}

Volume 15, numéro 2, 2e semestre 2002

Censure et traduction dans le monde occidental

Censorship and Translation in the Western World

URI : https://id.erudit.org/iderudit/007487ar

DOI : https://doi.org/10.7202/007487ar

Aller au sommaire du numéro

Éditeur(s)

Association canadienne de traductologie

ISSN

0835-8443 (imprimé)

1708-2188 (numérique)

Découvrir la revue

Citer ce compte rendu

Tatilon, C. (2002). Compte rendu de [Katharina Reiss. La critique des traductions, ses possibilités et ses limites, traduit de l'allemand par Catherine Bocquet, Cahiers de l'Université d'Artois 23/2002, Arras, Artois Presses Université, 2002, 166 p.] TTR, 15(2), 235-239. https://doi.org/10.7202/007487ar d'utilisation que vous pouvez consulter en ligne.

https://apropos.erudit.org/fr/usagers/politique-dutilisation/ 


\section{COMPTES RENDUS}

Katharina Reiss. La critique des traductions, ses possibilités et ses limites, traduit de l'allemand par Catherine Bocquet, Cahiers de l'Université d'Artois 23/2002, Arras, Artois Presses Université, 2002, $166 \mathrm{p}$.

Le but de cette étude, publiée en 1971 à Munich, est de présenter un modèle méthodologique permettant « une critique objective des traductions »(p. 12). Ce qui revient à poser la question: "Comment savoir si une traduction est bonne ou mauvaise ? " (quatrième de couverture). Pour y répondre, bien entendu. En cent cinquante pages d'une analyse serrée.

Ce qui m'a surtout frappé à la lecture de Katharina Reiss, c'est le déroulement rigoureux de sa démarche théorique. Une démarche inscrite dans une perspective précise: celle de la « traduction normale », par laquelle " on tente d'élaborer en langue-cible un texte équivalent au texte-source sans rien retrancher, sans rien ajouter ni distordre » (p. 33) - forme canonique de traduction qui exclut toute « réexpression en langue-cible d'un contenu reçu en langue-source [ayant une] visée différente de celle du texte original, ou un nouveau public-cible, autre que celui de l'original » (p. 41).

Dans cette démarche, tout s'enchaîne en une suite logique de propositions : a) le texte à traduire s'inscrit dans une typologie " qui englobe la totalité des genres de textes que l'on rencontre dans la pratique » (p. 32); b) il correspond à un type précis — «à dominante informative ", "à dominante expressive » ou « à dominante incitative » c) ce type, à son tour, «détermine la nature de l'équivalence -»; laquelle penchera tantôt du côté de contenu, tantôt du côté de la forme (ces deux notions largement discutées et assez clairement définies). La typologie des textes apparaît donc comme la clé de voûte de tout l'édifice théorique : tout au long de l'ouvrage est développée l'idée d'un lien fort entre le type de texte de départ et la nature de l'équivalence à établir.

Tirée au cordeau, la présentation ci-dessus, soucieuse avant tout de dégager ce qui m'a semblé constituer les lignes de force de l'ouvrage, ne rend pas bien justice à la souplesse de pensée de 
Katharina Reiss, qui souligne à maintes reprises la rigidité de sa classification. Mais, rigueur oblige : une méthode se doit d'avoir des contours nets, sous peine de ne pas être utilisable : "Commencer par déterminer le type de textes dont relève le texte en cause et ce, en se fondant sur le texte original. Cette étape une fois franchie [...], on se demandera si le traducteur s'est conformé à la hiérarchie des éléments à conserver dans la version-cible. » (p. 68) Et, par la vertu d'une rédaction limpide, tout, du début à la fin de l'ouvrage, donne prise à une compréhension sans équivoque. Ce qui n'est pas une mince qualité pour un texte « informatif».

Les aspects de cette étude fouillée sont multiples, mais deux d'entre eux m'ont particulièrement attaché. D'une part, la classification proposée pour baliser la planète Texte - son élaboration, sa nature et l'usage qui en est fait; d'autre part, l'évaluation de la traduction d'une écriture singulière.

L'élaboration de cette classification commence par un vaste tour d'horizon des propositions faites antérieurement par certains traductologues pour répondre aux besoins particuliers de la traduction. Dix tentatives typologiques sont ainsi analysées (celles, entre autres, d'Andrei V. Fedorov et de Georges Mounin). Se fondant sur les travaux de Karl Bühler (Sprachtheorie, 1934) qui distingue trois fonctions du langage fondamentales, à savoir l'information (Darstellung), l'expression (Ausdruck) et l'incitation (Appell), Katharina Reiss propose sa propre classification, que sa traductrice française, Catherine Bocquet, présente ainsi, avec mention, pour chaque type, de la stratégie préconisée : " pour les textes à dominante informative (inhaltsbetont), qui sont des textes centrés sur l'objet dont ils traitent, il s'agit avant tout de faire passer le contenu ; pour les textes à dominante expressive (formbetont), qui sont centrés sur l'émetteur, il convient de se conformer aux préoccupations esthétiques de l'auteur de l'original afin de transmettre aussi la forme, alors que pour les textes à dominante incitative (appellbetont), qui sont centrés sur le récepteur, le traducteur s'efforcera de provoquer chez le lecteur de la version-cible les mêmes réactions que celles que visait à obtenir le texte-source pour le récepteur de la version originale. » (Présentation, p. 8)

Ce court résumé laisse clairement apparaître un dérapage terminologique. Centrés sur l'émetteur, les textes à dominante expressive font ici problème: ils reflèteraient prioritairement les préoccupations esthétiques (!) de leurs auteurs. L'erreur ne vient pas de 
Catherine, fidèle traductrice, mais bien de Katharina - et cette erreur n'est rien d'autre que le reflet de la confusion qui régnait encore dans les années soixante-dix autour de la notion d'expressivité, souvent confondue avec celle d'impressivité, voire (comme ici) avec des aspects purement esthétiques. La fonction expressive, chez Karl Bühler comme chez Nicolas Troubetzkoy et Roman Jakobson, est bien centrée sur l'émetteur, mais en cela précisément qu'elle renseigne sur l'état d'esprit de celui-ci, sur l'opinion qu'il " exprime " vis-à-vis de l'information référentielle qu'il émet — renseignant aussi parfois, à son insu, sur sa personne physique, sa personnalité, son appartenance sociale, son degré d'instruction, son origine géographique, etc. L'ambiguïté est patente chez Katharina Reiss entre un contenu (cette information caractérisant l'émetteur) et la forme linguistique que prend ce contenu.

Mon objection va bien au-delà d'une chicane terminologique car le glissement sémantique brouille la précieuse et délicate analyse de l'information artistique et, partant, compromet la traduction des « éléments à conserver » (toutes les fois qu'ils sont esthétiquement pertinents) d'un texte à traduire. Confusion regrettable. On ne trouvera pas dans l'ouvrage la mise au point définitive qu'on attend encore en matière de «style ». Poussé depuis des siècles dans le sens de la montée, le rocher théorique a encore bien des tours à faire avant d'atteindre les derniers mètres de l'ascension. Au moins, chez Reiss, le débat est-il stimulant. Et, par les nombreuses réserves qu'il suscite, il nous incite à raffiner la problématique, à rechercher d'autres voies que celle ici proposée.

D'accord, donc, pour trois types majeurs. D'accord aussi, en principe, pour nommer ces trois types informatif, incitatif et expressif. Mais à condition de s'en tenir, pour le dernier cité, au sens bühlérien du terme (expressif: qui exprime l'opinion, les sentiments - Roman Jakobson parle aussi de fonction émotive - la personnalité, les origines de l'auteur - et Pierre Léon propose, au pluriel, des fonctions identificatrices); à condition aussi de convenir que le contenu expressif ainsi défini peut avoir, selon les textes, une certaine pertinence (à déterminer toujours en se référant à la finalité du texte d'arrivée). Mais alors, une question peut se poser: trouvera-t-on beaucoup de textes «expressifs »? Sans doute: tous les textes d'opinion, politiques, journalistiques ou autres. Mais encore : la plupart de ces textes ne sontils pas de bons candidats pour la catégorie « incitative »? Et surtout, comment pourrait-on faire l'impasse sur les textes littéraires, catégorie 
attestée depuis l'Antiquité et ressortissant à une communication de type très particulier ? Par ailleurs, ne faut-il pas traiter le problème de la forme séparément et au coup par coup? (Quelle est son rôle dans la formulation d'un contenu pertinent? A-t-elle une valeur esthétique?). $\mathrm{Ne}$ perdons pas de vue que ce redoutable problème formel se pose inlassablement (avec des degrés de complexité certes différents) pour chaque texte à traduire, quel que soit le type auquel il appartient. Ce qui nous amène au second aspect pris en compte par mon compte rendu, celui de l'écriture. (Ici, une précision terminologique : je désigne par le terme écriture la forme donnée à un texte particulier, et par style les traits de style récurrents qui caractérisent l'ensemble des écrits d'un auteur - écriture d'un texte, style d'un auteur.)

Quant à la bonne façon d'évaluer la traduction d'une écriture particulière, elle découle d'une préoccupation émanant de la perspective arrêtée, celle de la «traduction normale »: il convient de toujours " mesurer [une] traduction à l'aune du texte original, c'est-àdire [de toujours] établir la comparaison entre les deux. " (p. 16) Katharina Reiss préconise donc une "critique fondée sur le texte original ». (pp. 31-32) On retrouve ici la rigueur théorique mentionnée au début. Et ce précepte du retour au texte de départ, valable également pour l'autocritique que doit s'imposer tout traducteur digne du nom, peut être érigé en principe pédagogique : s'écarter de la norme de la langue d'arrivée n'est pas forcément une erreur - c'est même une nécessité dans le cas de textes manifestant une écriture singulière. «Le traducteur a parfaitement le droit de 'dépayser le lecteur' sans pour autant le dérouter, à moins que telle ne soit précisément la volonté de l'auteur de l'original. » (p. 56) Que de fois, lors de discussions académiques (voire à l'occasion de soutenances universitaires), n'a-ton pas vu afficher (parfois avec force) des opinions qu'on pourrait dire « antitraductologiques », fondées sur la base du seul texte d'arrivée et condamnant des « faiblesses [...] reproduites par souci de probité intellectuelle [et] immanquablement attribuées au traducteur et non pas à l'auteur du texte original »! (p. 87) Traduire la spécificité d'une écriture singulière est souvent un impératif, qui concerne - d'abord, certes, mais non exclusivement - le texte littéraire, engagé dans une recherche quasi permanente de l'inattendu. Souvenons-nous de la belle formule anthropologique de Stéphane Mallarmé, « donner un sens plus pur aux mots de la tribu », sur laquelle René Char renchérit en laissant percevoir des échos provenant des lointaines origines de l'Humanité : «Il faut réapprendre à frapper le silex à l'aube, s'opposer au flot des mots. » 
C'est pourquoi, retournant à la question typologique des trois types fondamentaux, je préfère, d'une part, substituer texte littéraire à texte expressif et, d'autre part, considérer l'expressivité comme un contenu informant sur l'auteur du texte (intentionnellement: ce qu'il écrit pour exprimer sa réaction personnelle au sujet traité dans son texte, ou involontairement : ce qui transparaît de lui dans le texte, par exemple son origine sociale ou géographique). Un contenu dont il faut, comme toujours, évaluer la pertinence à partir de la finalité accordée au texte d'arrivée.

Appliquée à La critique des traductions, la méthode de Katharina Reiss conduit à une évaluation élogieuse du travail de sa traductrice, Catherine Bocquet, qui a su rendre avec beaucoup de lisibilité une étude restée pendant trente ans " lettre close pour les francophones non germanistes ", n'hésitant pas à l'actualiser et à la naturaliser par d'abondants commentaires (une quarantaine de [NdT] sur près de 300 notes de bas de page).

Si cette étude, par la somme des connaissances qu'elle contient ainsi que par la rigueur de ses analyses, s'adresse bien en priorité aux spécialistes, je voudrais cependant suggérer qu'elle pourra convenir tout aussi bien aux traducteurs novices, auxquels elle rendra l'inestimable service de les conduire à plus de lucidité devant les nombreux obstacles qu'on rencontre dans la pratique de la traduction.

Claude Tatilon, Collège Glendon, Université York

Lynne Bowker. Computer-Aided Translation Technology: A Practical Introduction, Ottawa, Didactics of Translation Series, University of Ottawa Press, 2002, 185 p.

When I have a question about computer applications and translation and I need a quick and dirty answer, I call Lynne Bowker. Like other teachers of translation and practising translators, I have come to respect her understanding of the technologies and the pros and cons of integrating them into a translation program. This is not in itself a textbook for use in a course on computer aids to translation, though it could probably serve that purpose: it is, as it advertises itself, an introduction to the products and applications that translators may need 